\title{
Empleo del penetrómetro para madera para el diagnóstico de la madera en servicio en edificios antiguos
}

\section{Use of wood penetrometer for the diagnosis of timber structures in service, in old buildings}

\author{
L. Palaia ${ }^{(*)}$ \\ RESUMEN
}

Las técnicas instrumentales para ensayos in situ permiten a los expertos ahorrar tiempo en el diagnóstico y minimizar el daño que se produce en algunos casos a la madera sana, empleando técnicas tradicionales de diagnóstico. El empleo del Pilodyn para ensayos de penetración puede constituir un «método rápido» de diagnóstico de estas estructuras. Se presenta un caso de estudio de un edificio del siglo XIX construido en el centro histórico de Valencia. Los muros son de ladrillo y la estructura horizontal así como de la cubierta son de madera. Se ha realizado un método de diagnosis simplificado: inspección visual de la estructura y ensayos con Pilodyn. Este método resultó adecuado para determinar parámetros acerca de sus cualidades resistentes, abriendo una vía hacia una posible propuesta de normalización para el empleo de este instrumento.

Palabras clave: Estructuras de madera; diagnosis; edificios antiguos; Pilodyn.

\section{SUMMARY}

Instrumental technique applied in situ allows researchers to save time on diagnosis and minimize damage to the sound timber. Pilodyn testing may be a useful method of diagnosis of these structures. The case study is about an urban 19th century building in an historic area of Valencia, located very close to the original city foundation area. The building has brick bearing load walls, timber floors and a roof structure. A simplified diagnosis method of timber structures was performed: visual inspection carried out by qualified site surveyors using SDT, in this case the wood probing (Pilodyn). This method proved to be adequate to determine parameters and to obtain conclusions of the mechanical characteristics of the structural elements. A standardization proposal to use this instrumental may be developed in the future.

Keywords: Timber structures; assessment; historic buildings; Pilodyn.

(*) Universidad Politécnica de Valencia, España.

Persona de contacto/Corresponding author: lpalaia@csa.upv.es (L. Palaia)

Cómo citar este artículo/Citation: Palaia, L. (2014). Empleo del penetrómetro para madera para el diagnóstico de la madera en servicio en edificios antiguos. Informes de la Construcción, 66(533): eoo3, doi: http://dx.doi.org/10.3989/ic.12.055.

Licencia / License: Salvo indicación contraria, todos los contenidos de la edición electrónica de Informes de la Construcción se distribuyen bajo una licencia de uso y distribución Creative Commons Reconocimiento no Comercial 3.o. España (cc-by-nc). 


\section{INTRODUCCIÓN}

La mayoría de los edificios históricos tienen elementos y sistemas constructivos realizados con madera en las estructuras que soportan la cubierta y los forjados, que suelen presentar señales de degradación, algunas veces visibles y, otras veces, inapreciables a simple vista. Este material es susceptible de presentar ataques de hongos y de insectos. Una vez que se ha iniciado la alteración, esta continúa extendiéndose si las condiciones son las adecuadas. Sin embargo, la patología en las estructuras antiguas puede tener múltiples orígenes. No siempre es debida a causas biológicas, sino que pudo estar ocasionada por que la madera no ha sido introducida en el edificio cuando estaba bien seca, su calidad no era muy buena, o por que los carpinteros que las construyeron no eran tan buenos como se esperaba de ellos. Su falta de mantenimiento durante su vida útil así como el incremento de las sobrecargas de uso debido al cambio funcional al que se destina el edificio, pueden ser también causas de una reducción de las prestaciones de esas estructuras.

En su restauración y consolidación estructural, la evaluación del deterioro es importante cuando se decide sobre el tipo de trabajos de reparación necesarios y el coste de éstos. No hay que olvidar que la estructura pertenece a un edificio y éste, seguramente, habrá tenido una evolución desde los orígenes de su construcción, con ampliaciones, reformas, épocas de abandono y refuerzos estructurales. Es imprescindible para el diagnóstico de una estructura contar con estos datos, así como definir su geometría, identificando los elementos originales, y buscar una justificación a las reparaciones que puedan haber tenido lugar. Por lo tanto, para realizar el diagnóstico de una estructura de madera se han de tener en cuenta los siguientes aspectos:

- sistema de construcción original y detalles constructivos

- reparaciones anteriores

- calidad de la madera

- procesos de alteración

- tratamientos previos aplicados a la madera protectores contra la humedad

- tratamientos previos preventivos de ataques de hongos y de insectos

- movimientos estructurales

- criterios erróneos para reutilizar los edificios históricos

Para este fin, se pueden emplear los métodos tradicionales de inspección in situ pero muchos de ellos incluyen procedimientos que dañan al elemento de madera y destruyen parcialmente las fábricas que lo rodean. Existen otros métodos aplicables al diagnóstico de estas estructuras, que incluyen ensayos no destructivos (NDT) o semi-destructivos (SDT) realizados tras una inspección visual, reduciendo o eliminando en algunos casos, el empleo de ensayos destructivos.
En este artículo se presenta un método simplificado de diagnóstico de estructuras de madera mediante el empleo del penetrómetro para madera Pilodyn. La elaboración del método que se propone va más allá del trabajo de investigación realizado "Aplicación de métodos no destructivos para inspección y diagnóstico de materiales y sistemas constructivos en edificios históricos para su conservación ${ }^{1}$, que incluía el diagnóstico de la madera. Se aplicaban distintas técnicas, como la auscultación ultrasónica, el resistógrafo para madera y el penetrómetro Pilodyn (1).

Se expondrán los antecedentes en el empleo del penetrómetro Pilodyn y se incluye una experiencia en la que se podrá comprobar la eficacia y fines del mismo.

\section{ANTECEDENTES}

El aspecto a destacar en el empleo de estos métodos de diagnóstico es la realización de ensayos in situ a fin de determinar de una manera no solo cualitativa sino también cuantitativa, el estado de la estructura. Después de la inspección visual, paso inicial de todo proceso de diagnosis, el instrumental que se puede emplear, dentro de los NDT o SDT, generalmente, es el siguiente (2) (3) (6):

- Ultrasonidos, para la aproximación al módulo de elasticidad (4)

- Penetrómetro para madera "Pilodyn 6J, para la aproximación a la densidad y consistencia superficial (6)

- Resistógrafo, para observar el interior de los elementos lígneos (5) (6)

- Arranque de tornillos, como método de aproximación a la densidad de la madera (6)

- Xilohigrómetro, para determinar el contenido de humedad de la madera

Si bien el empleo de estas tecnologías se dirige a cuantificar datos con respecto a la resistencia mecánica de la madera, también es cierto que el empleo de todas ellas para un estudio concreto se puede simplificar, cuando las necesidades o características del inmueble así lo requieran. Una posible simplificación del método de inspección consiste en el empleo del Pilodyn tras la realización de una inspección visual de la estructura, permitiendo efectuar un diagnóstico que se puede denominar «rápido», no por ello carente de rigor metodológico, cuyo propósito es corroborar las conclusiones obtenidas en la inspección visual.

\subsection{Empleo del penetrómetro para madera}

El penetrómetro para madera, conocido por su marca comercial Pilodyn, consiste en una aguja de acero de $2,5 \mathrm{~mm}$ de diámetro y 60,2 $\mathrm{mm}$ de longitud, que se dispara en la madera con una energía constante y, a partir de la penetración de la misma, se puede estimar su densidad o el grado de alteración que presenta (7). Fue diseñado en Suiza a fin de de-

\footnotetext{
${ }^{1}$ El equipo de investigación estaba formado por Liliana Palaia ${ }^{1}$, José Monfort² ${ }^{2}$ Rafael Sánchez ${ }^{3}$, Luisa Gil ${ }^{4}$, Ángeles Álvarez ${ }^{5}$, Vicente López ${ }^{5}$, Santiago Tormo ${ }^{5}$, Carmen Pérez 6 , Pablo Navarro $7{ }^{7}$ Catedrática de Construcciones Arquitectónicas ${ }^{2}$, Catedrático de M M C y Teoría de las Estructuras, ${ }^{3}$ Profesor Titular de Física Aplicada, ${ }^{4}$ Profesora Titular del Departamento de Construcciones Arquitectónicas, ${ }^{5}$ Profesores Asociados del Departamento de Construcciones Arquitectónicas, ${ }^{6}$ Catedrática del Departamento de Conservación de Bienes Culturales, ${ }^{7}$ Catedrático de Expresión Gráfica, todos ellos de la Universidad Politécnica de Valencia (2007). Proyecto financiado por el Ministerio de Fomento, Convocatoria 2004.
} 
terminar el grado de pudrición en postes telefónicos (8) (9). La penetración de la aguja es inversamente proporcional a la densidad o estado de conservación de la madera, teniendo importancia su contenido de humedad, puesto que su mayor o menor contenido afecta a las propiedades físicas del material. La desventaja del método es que solamente se pueden medir características superficiales del material, hasta $40 \mathrm{~mm}$ de profundidad.

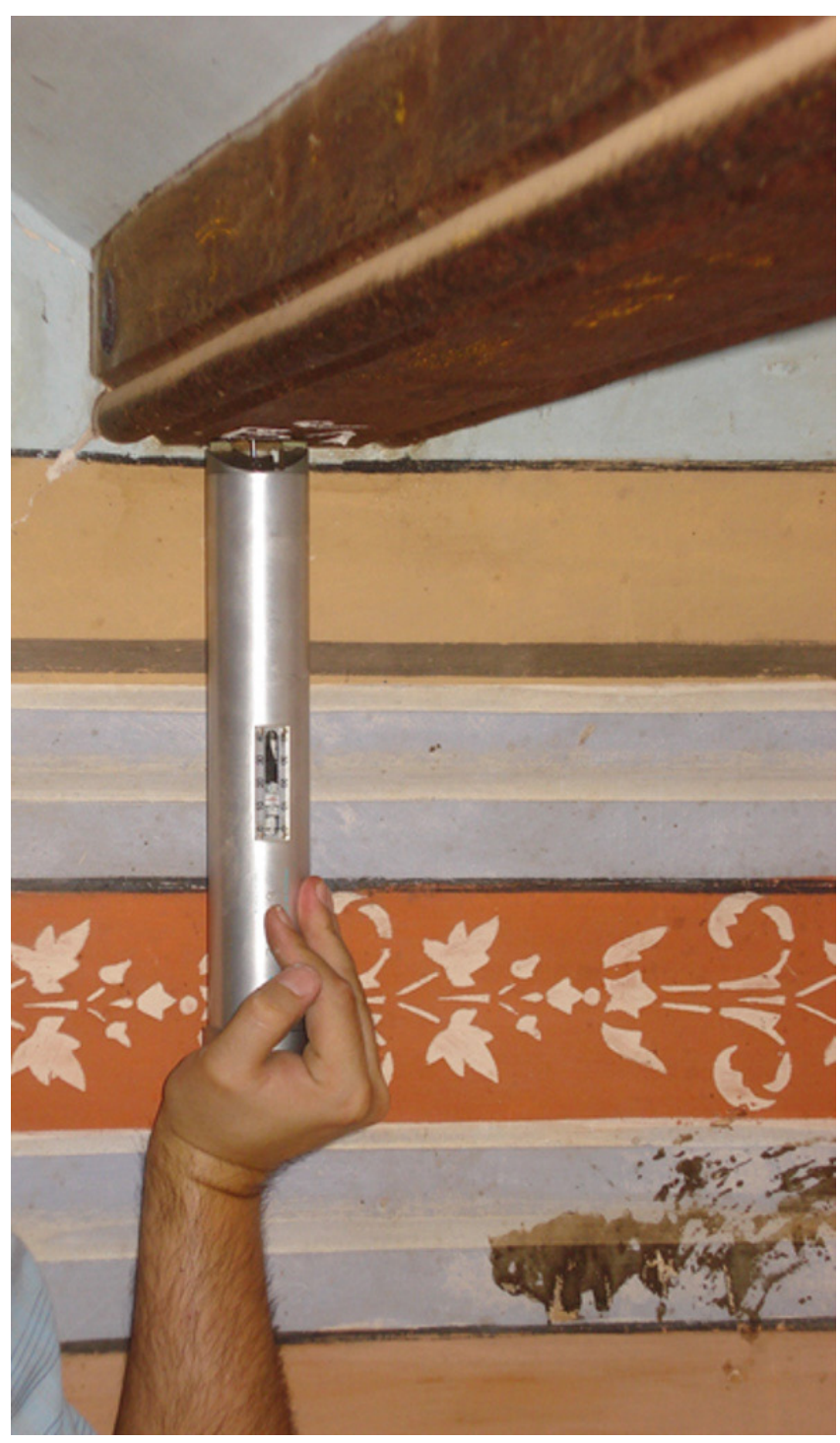

Figura 1. Empleo del penetrómetro Pilodyn 6J.

El modelo utilizado es el Pilodyn 6J (Figura 1), que detecta la consistencia de la madera, dando valores «normales» de penetración de la aguja entre 9 y $12 \mathrm{~mm}$, pudiendo llegar a 13, dependiendo de las especies, mientras que si este valor aumenta podría indicar que se ha detectado un estado de pudrición o algún otro tipo de alteración. Los datos técnicos del instrumento son los siguientes: Energía de impacto: $6 \mathrm{~J}$ (Nm), Profundidad de penetración: 0-40 mm, Varilla percutora: 2,5 $\mathrm{mm}$ de diámetro, Dimensiones del equipo: cuerpo $270 \mathrm{~mm}$ de longitud (con aguja en extensión $320 \mathrm{~mm}$ ) y 50 $\mathrm{mm}$ de diámetro. Según se aprecia en estos datos y en la imagen superior, se trata de un instrumento fácilmente transportable que ofrece ese aspecto como una de sus principales ventajas. Es, por lo tanto, un instrumento ligero que puede evaluar las cualidades de la madera en capas superficiales y que según el tipo de diagnóstico que se pretenda realizar puede dar resultados cuantificables de la calidad de la madera en un área determinada (Local Test Method, LTM) así como de su estado de conservación.

Los datos que se obtienen hay que correlacionarlos con los que aportan otros SDT y NDT como el resistógrafo, arranque de tornillos o los ultrasonidos, o bien con resultados de ensayos realizados con métodos destructivos. En el caso de no contar con datos específicos para la especie de madera empleada en el edificio, se puede trabajar con los resultados de manera comparativa.

El penetrómetro para madera se emplea desde hace varias décadas tanto en la evaluación de árboles en pie como en el diagnóstico de la madera en servicio.

Las primeras investigaciones realizadas con este instrumento estaban destinadas a establecer un método rápido para la determinación de la densidad de la madera de árboles en pie de Pinus taeda que se recogen en el artículo de Taylor (9), basados a su vez en las experimentaciones de Hoffmeyer de 1978 (10) en el que se introducía el empleo del Pilodyn como NDT, para predecir la resistencia de la madera de Abies Alba. En ese mismo año, Cown (11) evaluaba este penetrómetro como método para determinar la densidad de la madera de Pinus radiata. Todos estos artículos estaban dirigidos a establecer clases resistentes en los árboles en pie, como una preselección de éstos a la hora de su aprovechamiento y mejora de su producción. En el artículo de Taylor antes mencionado, se establecía que en Pinus taeda, penetraciones de $14 \mathrm{~mm}$ correspondían a árboles con densidad elevada mientras que las penetraciones de $24 \mathrm{~mm}$ lo eran para madera de baja densidad. En todos ellos se realizaban lecturas de resistencia a la penetración y ensayos destructivos con muestras obtenidas de los árboles en pie, de madera de albura. La correlación de resultados estaba en $r=0,81$, siendo estadísticamente relevante.

Numerosos son los artículos publicados con este fin, cada vez más específicos (12) (13) (14) (15) en los cuales el propósito era mejorar el grado de crecimiento de los árboles sin perder cualidades características de la madera, que se basaban principalmente en la determinación de su densidad. Sin embargo, Hansen (8) indica que los resultados del Pilodyn no determinan la densidad de la madera, sino que la estiman, por comparación de los resultados obtenidos de los ensayos realizados en una misma especie. Incluye un listado de aplicaciones - situándonos en el año 2000 - en distintas especies para mejorar la calidad de producción de madera. Se incluye la tabla según Hansen (Tabla 1).

En otros trabajos se emplea este instrumento junto con determinaciones del módulo de elasticidad dinámico en madera de alerce, por medio de trasmisión de ondas provocadas por impacto (stress-wave velocity) (14), contrastado con ensayos destructivos, determinando que existe una correlación inversa entre el módulo de elasticidad estático y las lecturas realizadas mediante Pilodyn.

Las investigaciones realizadas para el diagnóstico de la madera en servicio parten de los ensayos de dureza superficial del material como indicativo de sus cualidades mecánicas. 
Tabla 1.

Aplicaciones del Pilodyn para mejora del crecimiento de distintas especies.

\begin{tabular}{|c|c|}
\hline Especies & Autor(es) \\
\hline Acacia aulacocarpa & Sim and Gan (1991) \\
\hline \multicolumn{2}{|l|}{ A. auriculiformis } \\
\hline \multicolumn{2}{|l|}{ A. crassicarpa } \\
\hline \multicolumn{2}{|l|}{ A. mangium } \\
\hline \multicolumn{2}{|l|}{ A. mearnsii } \\
\hline Gmelina arborea & Lauridsen et al. (1983); Akoun et al. (1985) \\
\hline Eucalyptus sp. & Chapola (1994) \\
\hline Eucalyptus camaldulensis & Moura et al. (1987) \\
\hline Eucalyptus globulus ssp. globulus & MacDonald et al. (1997) \\
\hline Eucalyptus grandis & Vale et al. (1995) \\
\hline Eucalyptus nitens & Greaves et al. (1996) \\
\hline Eucalyptus urophylla $x$ E. grandis & Bouvet and Vigneron (1996) \\
\hline Fagus sylvatica & Lewark and Giefing (1983) \\
\hline \multirow[t]{2}{*}{ Picea abies } & Gorlacher (1987); Lewark and Giefing (1983); \\
\hline & Piroton et al. (1995) \\
\hline Picea mariana & Antal and Micko (1994) \\
\hline Picea sitchensis & Costa E. Silva et al. (1994); Hansen and Roulund (1997) \\
\hline Pinus caribaea & Moura and Parca (1993) \\
\hline Pinus engelmannii & Yanchuk and Kiss (1993) \\
\hline Pinus elliottii & Gough and Barnes (1984) \\
\hline Pinus glauca & Yanchuk and Kiss (1993) \\
\hline Pinus oocarpa & Wright et al. (1992) \\
\hline Pinus patula ssp. tecunumanii & Moura and Parca (1993); Wright et al. (1992) \\
\hline Pinus pinaster & Notivol et al. (1992) \\
\hline Pinus radiata & Cown (1982); Watt et al. (1997) \\
\hline Pinus sylvestris & Haapanen et al. (1997) \\
\hline Pinus taeda & Sprague et al. (1983); Taylor (1981) \\
\hline Pseudotsuga menziesii & King et al. (1988) \\
\hline
\end{tabular}

En los años 90 del siglo pasado, en estudios de A. M. Korzeniowski y Dzbenski (15) se efectuaron ensayos de dureza por impacto para predecir las principales características de la madera, relacionadas con su resistencia mecánica. Los ensayos normalizados se realizaron al $12 \%$ de contenido de humedad para la determinación de la densidad, resistencia a la flexión, compresión, tracción, resistencia a la cizalladura y el módulo de elasticidad, tanto en duramen como en albura, en probetas de madera de pino (Pinus silvestrys L.) limpias de defectos. Se comprobó la dureza por medio del aparato de Baumann modificado, diseñándose un durómetro portátil con una energía de impacto de 6,67 Nm. Se verificaron relaciones entre la dureza y las propiedades mecánicas, permitiendo recomendar el ensayo de dureza por impacto no destructivo para clasificar las maderas estructurales.

Otro investigador, A. Gubana, publicó en esas mismas fechas, varios artículos con otros autores (16) (17) (18) en los que desarrollaba un método de caracterización mecánica de la madera en servicio mediante un penetrómetro diseñado por el equipo de investigación, basado en la penetración de una varilla graduada de $3 \mathrm{~mm}$ de diámetro, que avanzaba por medio de golpes repetidos de un martillo de rebote de
2,2 J de energía constante. En los primeros trabajos se buscó encontrar una correlación entre la resistencia a flexión sobre un prisma de madera de $2 \times 2 \times 30 \mathrm{~cm}$ según ISO 3133 (19) y los resultados del ensayo de penetración, dando un resultado fiable la curva de correlación obtenida (16). Las conclusiones del trabajo indican que el sistema es simple y no destructivo, y que sirve para clasificar la calidad resistente de tablas de coníferas, pero no justifica sus defectos estructurales de influencia contraria a las propiedades mecánicas de la madera. Esta técnica se aplicó en una investigación de la bóveda del techo del Palazzo della Loggia, una construcción del siglo XVI de Brescia (Italia), y dio unos resultados muy útiles acerca del alcance y profundidad del deterioro de la estructura resistente de madera. A. Gubana con P. Ronca (18) muestran una campaña de ensayos con probetas de madera de abeto rojo, tanto de dureza como de resistencia a flexión, y con contenidos de humedad del 12, 15, 18, 25 y 30\%. Entre las conclusiones de la experimentación se destaca que se dan las mejores correlaciones de resultados resistencia mecánica-número de golpes, al 12\% de contenido de humedad, en dirección radial, situación que se mantiene si la inclinación de la tangente a los anillos respecto a la radial no es superior a $30^{\circ}$, siendo algo mayor la dispersión cuando el ensayo de penetración se 
realiza en dirección tangencial. Según los resultados de su experimentación, cuando la madera está saturada (más del 30\% de contenido de humedad) la diferencia entre los valores de penetración de la aguja en las direcciones radial y tangencial está en el orden del 5-6\%, pudiendo alcanzar una diferencia del 20\% para contenidos de humedad menores.

En trabajos más recientes Ignacio Bobadilla y otros investigadores (6) realizaron un estudio para estimar la densidad de la madera aserrada de conífera mediante el empleo de extractor de tornillos y penetrómetro Pilodyn 6J, a fin de estimar el módulo de elasticidad. Los ensayos se realizaron en maderas de Pinus radiata, Pinus sylvestris y Pinus nigra. La aplicación del penetrómetro se realiza en direcciones radial y tangencial, previa medición del contenido de humedad mediante xilohigrómetro y determinación de la densidad según normas europeas UNE-EN 408:2004 Estructuras de madera. Madera aserrada y madera laminada encolada para uso estructural. Determinación de algunas propiedades físicas y mecánicas (20) y UNE-EN 13183:2002; err 2003; Ac 2004. I-II Contenido de humedad de una pieza de madera aserra$d a$ (21). Una de las conclusiones del empleo del penetrómetro es que existen diferencias significativas de penetración de la aguja según las especies: la penetración media en Pinus radiata ha sido de 10,06 mm, en Pinus sylvestris de 10,78 y en Pinus nigra, 9,62 mm, correspondiendo a valores medios de densidad de 481, 500 y $586 \mathrm{~kg} / \mathrm{m}^{3}$ respectivamente. Los datos de penetración radial y tangencial se han considerado haciendo una media aritmética de los resultados de la medición. Teniendo en cuenta los dos métodos empleados en la investigación, los resultados subestiman la densidad de la madera en un $10 \%$ que quedaría, en todo caso, dentro del lado de la seguridad. Las mediciones las realizan en madera sana y limpia de defectos para no introducir más variables en los resultados.

Investigadores como M. Piazza y M. Riggio realizaron trabajos a fin de determinar la eficiencia de distintos NDT para la caracterización de estructuras de madera antiguas (22) (23) (24). En uno de estos trabajos (22) realizan indicaciones para aumentar la efectividad en la clasificación visual de la madera y el nivel de precisión de algunos NDT. Para la determinación de la densidad de los elementos estructurales emplean el Pilodyn, basándose en los estudios de V. R. Görlacher de 1987 (25), quien estableció su eficacia para predecir estos valores con razonable aproximación (coeficientes de correlación de 0,74 a 0,92), teniendo influencia el contenido de humedad de la madera. Señalan que el empleo del Pilodyn $6 \mathrm{~J}$ es eficaz para definir áreas con alteraciones, permitiendo realizar un mapeo de las mismas en los elementos estructurales, que requieren posteriores prospecciones por medios más sofisticados, como el empleo del resistógrafo, a fin de acotar la sección residual del elemento. En otro trabajo, el profesor Piazza (26) junto con otros investigadores de la universidad de Minho, analizan dos cerchas de madera mediante NDT y pruebas de carga, en los que incluyen el instrumento Pilodyn obteniendo medidas en las cuatro caras de los elementos estructurales, a fin de localizar zonas de menor densidad acotando posibles alteraciones del material. Los NDT empleados han sido la microperforación, ensayos de penetración, auscultaciones de ultrasonidos y evaluaciones mecánicas a fin de tener valores de módulo de elasticidad estático. Los datos de penetración comparados con los de densidad de la madera, han dado correlaciones lineales con coeficientes de determinación $\mathrm{R}^{2}$ de o,50, considerando los valores medios obtenidos en las me- diciones realizadas en las cuatro caras, con un contenido de humedad de alrededor del $12 \%$.

El profesor Schueremans (27) incluye entre los métodos de diagnóstico in situ de la madera, el empleo del Pilodyn para obtener información de la densidad y daño superficial de los elementos estructurales. Esta información provee datos «relativos», requiriéndose de otros ensayos para obtener datos cuantitativos.

Como conclusiones de estos trabajos cabe destacar lo siguiente:

- los métodos semi-destructivos dan información de una zona relativamente pequeña del elemento estructural (LTM)

- el penetrómetro para madera da buenos resultados cuando se quiere establecer relaciones con su densidad

- es conveniente tener en cuenta la dirección en la que se está introduciendo la varilla, puesto que puede variar los resultados

- es necesario establecer el contenido de humedad a la hora de realizar los ensayos in situ, por medio de un xilohigrómetro, dada la influencia del contenido de humedad en las características mecánicas de la madera

- existe, según los ensayos realizados y apoyados en estudios estadísticos, una diferenciación bastante significativa entre los resultados de ensayos de penetración en distintas especies de madera que han sido empleadas en la construcción de edificios históricos que permitirían una clasificación inicial que debería ser contrastada por medio de estudios anatómicos de laboratorio.

\section{APLICACIÓN DEL MÉTODO RÁPIDO DE DIAGNÓSTICO MEDIANTE EL EMPLEO DEL PILODYN. UN EDIFICIO DE LA CIUDAD DE VALENCIA DE FINALES DEL SIGLO XIX}

\subsection{Una metodología de diagnóstico}

La experiencia indica que la madera, por lo general, se encuentra en buen estado de conservación, excepto algunos elementos que se señalarían en los planos de planta o en alzados, para ser fácilmente identificados, a fin de ser reparados o reforzados, según el caso (3). Es una manera muy directa de plantear la intervención.

Realizada la inspección visual, se determina la clasificación según la clase resistente de acuerdo con la norma UNE 56544:2011. Clasificación visual de la madera aserrada para uso estructural. Madera de coníferas (28), así como la identificación de los daños detectados. A partir de este punto, la metodología de diagnóstico mediante el empleo de penetrómetro para madera que se presenta es adecuada para la obtención de datos aproximados de la densidad y consistencia superficial de la madera, confirmando las zonas de alteraciones localizadas en la inspección visual.

Dado que en las conclusiones de las investigaciones realizadas que se indican en el apartado 2.1, tiene importancia la dirección en la que se realiza el ensayo de penetración para determinar algunas de las características buscadas en el empleo de este SDT, indicaremos la casuística derivada de la experiencia recogida durante los estudios realizados de estructuras existentes en edificios antiguos en Valencia, para su evaluación. 
La comercialización de la madera escuadrada en Valencia se ha basado desde el siglo XIII hasta el XIX en el «marco valenciano» (29). Éste clasifica las maderas en grados según su escuadría y el largo de las piezas. El tocho o carga designa la unidad de volumen, identificada con el símbolo «A» con un largo de 30 palmos valencianos, 30 dedos de ancho y 26 dedos de canto. Todas las demás piezas se clasificaban con respecto a esta pieza de mayor sección, siendo las demás piezas clasificadas las siguientes: la mejoría con « $\mathrm{M} »$, la sisa con «U», el madero con «m», el cuaderno con «Q", seiseno con la «L», y el cabrio con la «V». A continuación siguen las filas, que se identifican con la «f» seguida con un número, que comienza en el 10, y aumenta a razón de cuatro hasta 30 y luego a razón de 6 , hasta 48 . Se puede comprender el marco valenciano a partir del cuadro que se presenta en la Figura 2 (30)

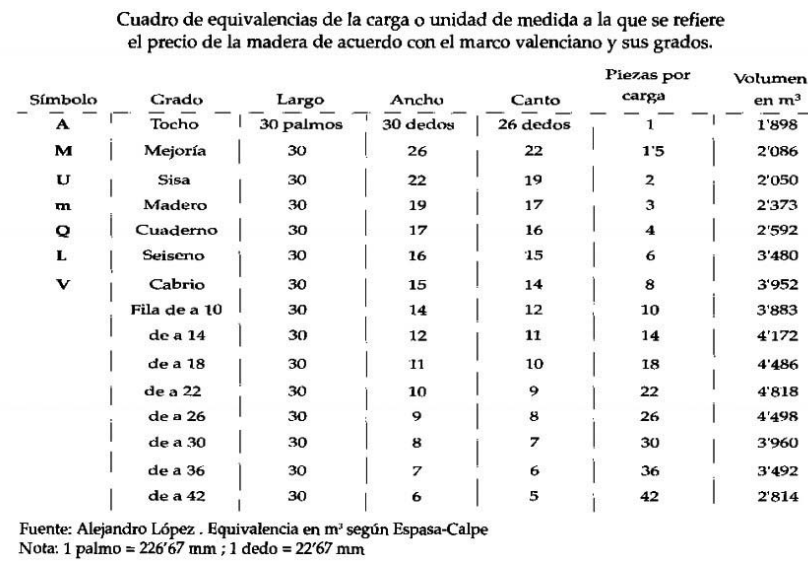

Figura 2. Cuadro explicativo del marco valenciano.

La madera que se empleaba como vigas eran las piezas comercializadas que se han enumerado anteriormente, dependiendo de las exigencias de servicio que estas tuvieran, es decir, si la sección y longitud fueran suficientes para la misión resistente a la que estaban destinadas (31). Se verifica que en los edificios residenciales, generalmente, las piezas empleadas como vigas eran cabrios de $14 \times 15$ dedos $(31,73 \times 34 \mathrm{~cm})$ o filas de a 18 de $10 \times 11$ dedos $(22,27 \times 24,93 \mathrm{~cm})$, siendo el dedo $2,267 \mathrm{~cm}$.

Para la formación de pares y de viguetas se partía de las piezas denominadas filas de a 18, con forma casi cuadrada, que el carpintero de armar las dividía por la mitad en su longitud, obteniendo piezas de entre 22 a $24 \mathrm{~cm}$ de altura, pero de menor base, más adecuadas para tal uso.

Cuando se empleaban como vigas, es decir fila de a 18 o bien cabrios, o cualquier otra pieza de sección y longitud suficiente, la disposición de los anillos en estos elementos tendría su centro coincidente con el centro geométrico de la sección de la madera escuadrada. En las diversas tomas de datos de estructuras in situ, los ensayos de penetración en vigas podrían realizarse tanto en dirección radial como tangencial, según su accesibilidad.

En las viguetas y en los pares se da otra circunstancia al ser el resultado de partir en dos una pieza escuadrada, teniendo el centro de los anillos coincidente con una de las caras de las viguetas. De esta manera, una de sus caras, que estaría cortada con sierra, presenta prácticamente duramen, mientras que la opuesta, que muestra las marcas de escuadrado con azuela, presenta albura en un espesor de unos 2-3 cm, ocurriendo lo mismo en las caras superior e inferior en ambos casos. Siendo así, solamente se podrían obtener lecturas radiales en viguetas y pares cuando el ensayo se realizara a mitad de la altura de las mismas, mientras que todas las demás posiciones serían ensayos a penetración en dirección tangencial, con inclinaciones con respecto a la radial de 30 a $40^{\circ}$ (Figura 3). Una clasificación de estos datos es posible, para su posterior análisis.
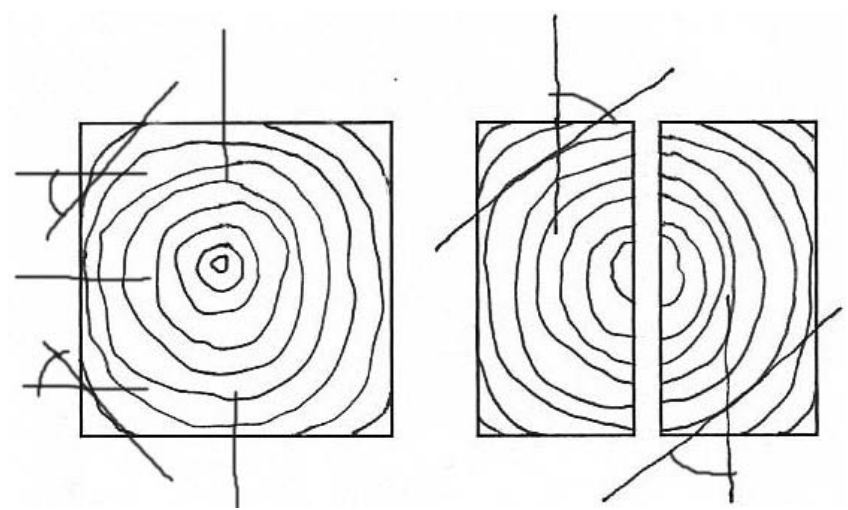

Figura 3. Configuración de los anillos anuales de crecimiento con respecto a la sección.

\subsection{Caso de estudio}

El edificio se encuentra en el barrio de Seu-Xerea, dentro del centro histórico de Valencia, a pocos metros del río Turia en la calle de El Salvador (Figura 4). Esta calle es una de las más antiguas de la ciudad, ya que muy cerca de ésta pasa el cardo de la ciudad romana, la Via Saguntina que unía Valentia Edetanorum con Saguntum, y se dirige hacia uno de los puentes del río Turia. En el siglo VIII la ciudad de Valencia fue conquistada por los árabes quienes permanecieron hasta la reconquista cristiana en el siglo XIII, construyéndose nuevas murallas en el siguiente siglo, que englobaron en su interior la zona donde está emplazado este edificio. La calle de El Salvador llevaba a una de las puertas que se abrían en la muralla, la Porta dels Catalans denominada así por comunicar con el camino que llevaba hacia el norte, o también de la Trinidad, por el convento que se encontraba a la otra orilla del río Turia.

En el siglo XVI, la zona delimitada por las actuales calles de El Salvador (antes de San Narciso) y de Libertad, y el río Turia, estuvo ocupada por la cárcel de San Narciso que fue ampliada en el siglo XVIII. Fue demolida al siglo siguiente dado que su capacidad se hacía insuficiente, trazándose nuevas líneas, variando poco las que siempre tuvo la calle de El Salvador, construyéndose edificios de viviendas de nueva planta a finales del siglo XIX.

De esa época data la construcción del edificio objeto de estudio, destinado a viviendas en alquiler, por lo que su configuración general es sencilla en cuanto a sus aspectos formales y a su sistema constructivo. Consta de planta baja, carece de entresuelo característico en edificios de mayor nivel social de la ciudad de Valencia en esos tiempos, y cuatro plantas de altura. La estructura vertical es de muros de ladrillo y la estructura horizontal y de cubierta, es de madera. 


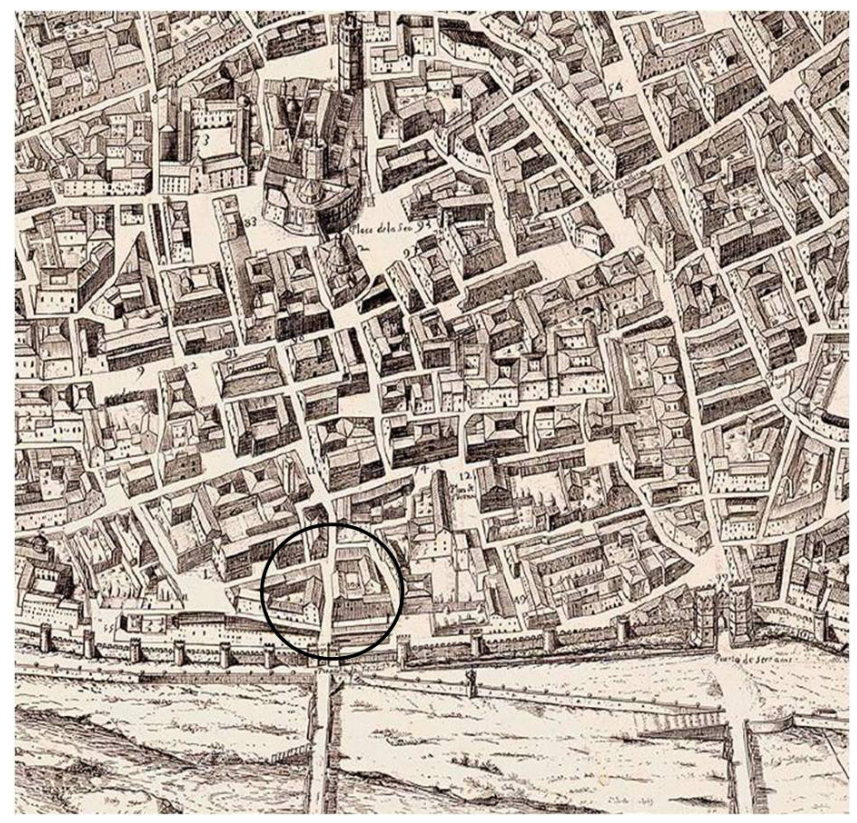

Figura 4. Área de la calle El Salvador, Plano 02. VALENTIA EDETANORUM ALIIS CONTESTATORUM/VULGO DEL CID. ICHNOGRAPHICE DELINEATa/a Dre. Thoma VinCENTIO TosCa Congreg. ORATORIJ PREFBYTERO-ANNO 1704 (32).

\subsection{El sistema constructivo}

El solar en el que se ha construido el edificio es de forma trapezoidal. Tiene tres crujías, dos de ellas regulares, siendo la de fachada, irregular. Las luces son normales para este tipo de construcciones, aunque presenta algunos elementos de longitud algo importante en la primera crujía (Figura 5). Todas las plantas tienen la misma composición de los forjados, formados por viguetas de madera y revoltones de ladrillo, y mantienen la misma disposición de viguetas. Asimismo, las vigas de madera sobre las que apoyan tienen aproximadamente las mismas escuadrías, de $30 \times 35 \mathrm{~cm}$ correspondiente al cabrio según el marco valenciano. Se han identificado como vigas 1, 2 y 3 dependiendo de su distancia al muro de fachada. Las viguetas tienen 4 elementos identificadores: 1 (nivel). 1 (número de crujía). 2 (número de vigueta). E y W (según el extremo), quedando del siguiente modo 4.1.3.E, lo cual indica forjado $4^{\mathrm{a}}, 1^{\mathrm{a}}$ crujía, vigueta 3 , extremo este. Las viguetas se han numerado comenzando por el extremo norte. Se puede apreciar la configuración de la estructura de la planta tipo en la Figura 5.

Varias de las viguetas parecen reutilizadas, encontrándose algunos elementos de mayor sección con escotaduras para apoyar en ellas los revoltones de ladrillos, mientras que otros elementos, de menor sección, tienen una lata o rastrel clavado a ambos lados de las viguetas, para cumplir la misma función. Las viguetas de la segunda crujía, en algunas plantas, están molduradas en las aristas. Algunas de las vigas y viguetas, que parecen estar formadas con madera más antigua, tienen una identificación en forma de «V» (que correspondería con el cabrio del marco valenciano), con una numeración, como se aprecia en las Figuras 6 y 7.

La estructura de la cubierta mantiene las tres crujías de las plantas inferiores (Figura 8). Las vigas de madera sobre las que apoyan los extremos oeste (W) de los pares de la primera crujía, y ambos extremos de los pares de las otras dos crujías,

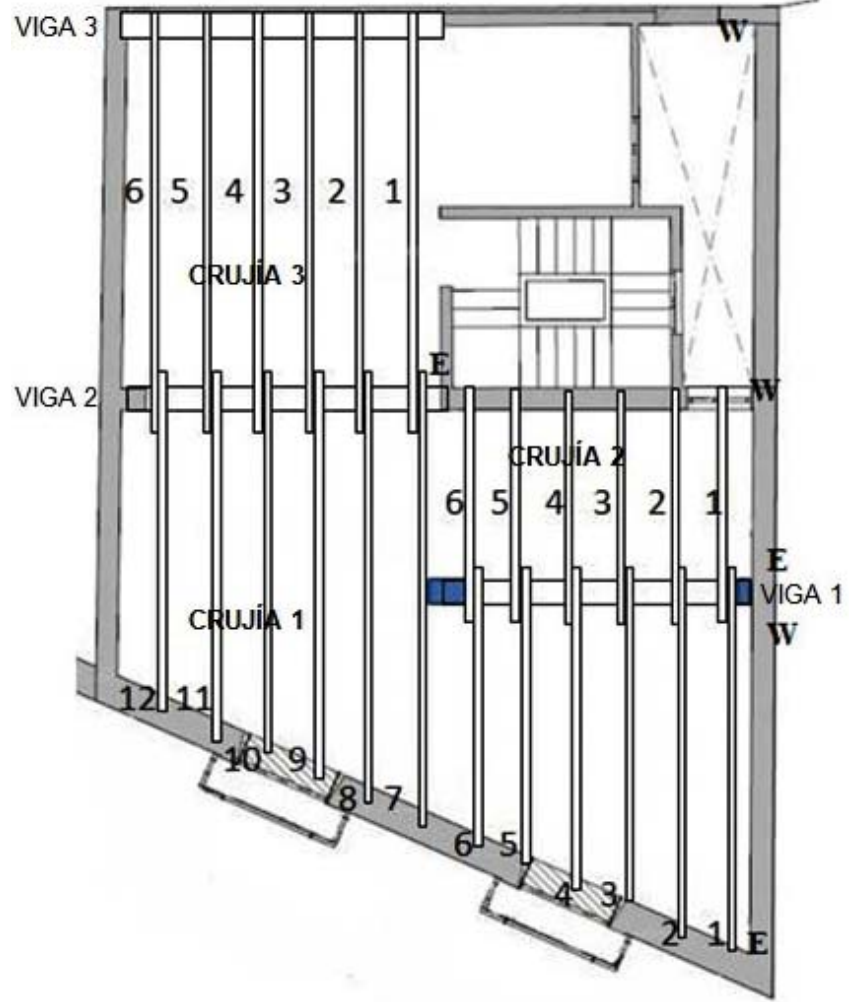

\begin{tabular}{|l|l|l|}
\hline VIGUETAS & $\begin{array}{c}\text { LONGITUD } \\
(\mathbf{m})\end{array}$ & $\begin{array}{c}\text { SEP. VIGUETAS } \\
(\mathbf{c m})\end{array}$ \\
\hline 4.1 .1 & $4,30 \mathrm{~m}$ & 70 \\
\hline 4.1 .6 & $2,70 \mathrm{~m}$ & \\
\hline 4.1 .7 & $5,39 \mathrm{~m}$ & \\
\hline 4.1 .12 & $3,80 \mathrm{~m}$ & \\
\hline $4.2 .1 \mathrm{a} 6$ & $2,60 \mathrm{~m}$ & 70 \\
\hline $4.31 \mathrm{a} 6$ & $4,30 \mathrm{~m}$ & 80 \\
\hline
\end{tabular}

Figura 5. Distribución de elementos lígneos y longitud de algunas de las viguetas. Planta tipo. Edificio c/ El Salvador.

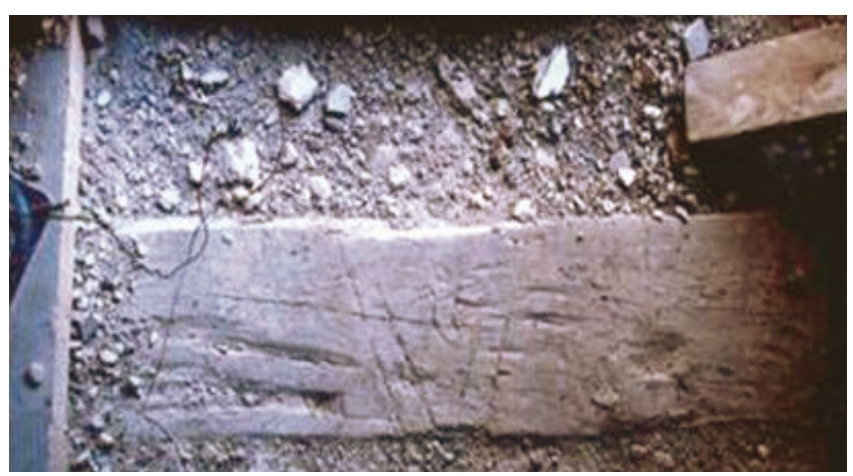

Figura 6. Vigueta 1.1.12. E.

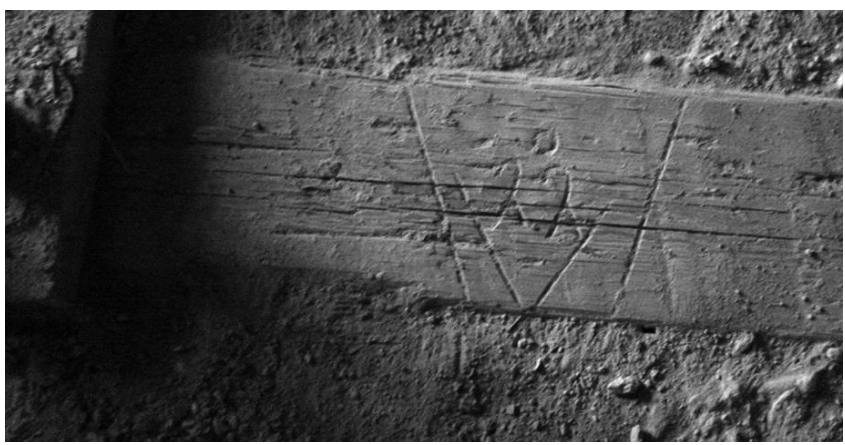

Figura 7. Vigueta en el forjado $4^{\mathrm{a}}$. 
son aproximadamente de las mismas dimensiones, $32 \times 34$ $\mathrm{cm}$. En la posición de la VIGA 2, que separa las crujías $1^{\mathrm{a}} \mathrm{y}$ $3^{\text {a }}$, hay dos vigas superpuestas de dimensiones similares que producen un salto en la altura de la cumbrera, siendo más alta la de la $3^{\text {a }}$ crujía (Figura 9).

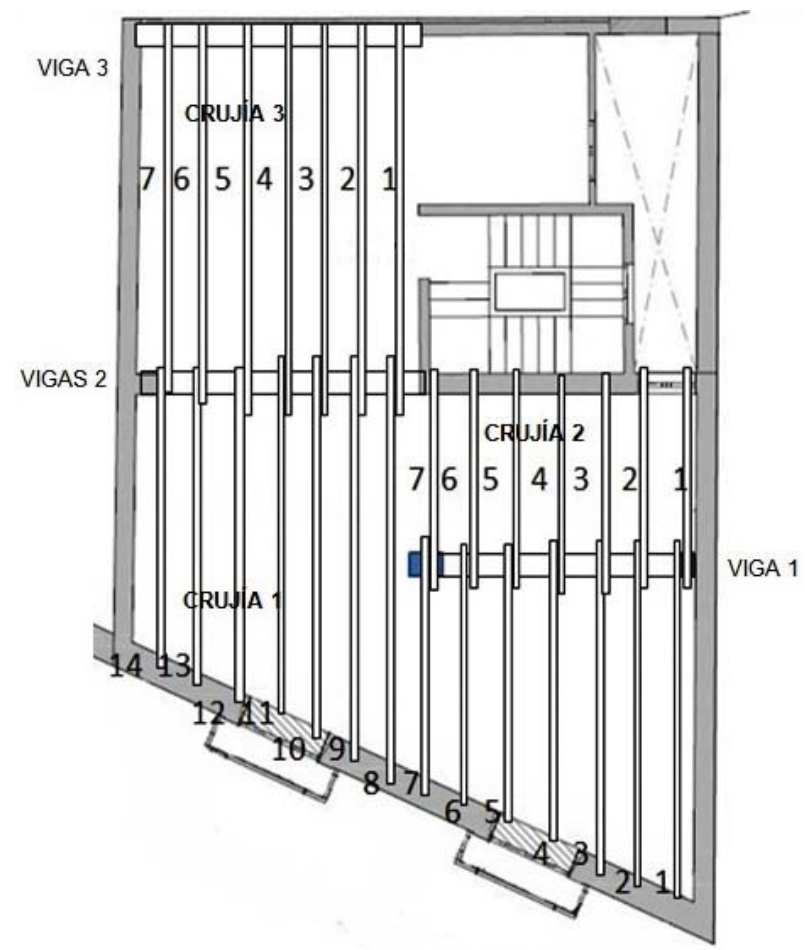

Figura 8. Distribución de elementos lígneos. Planta cubierta. Edificio c/ El Salvador.

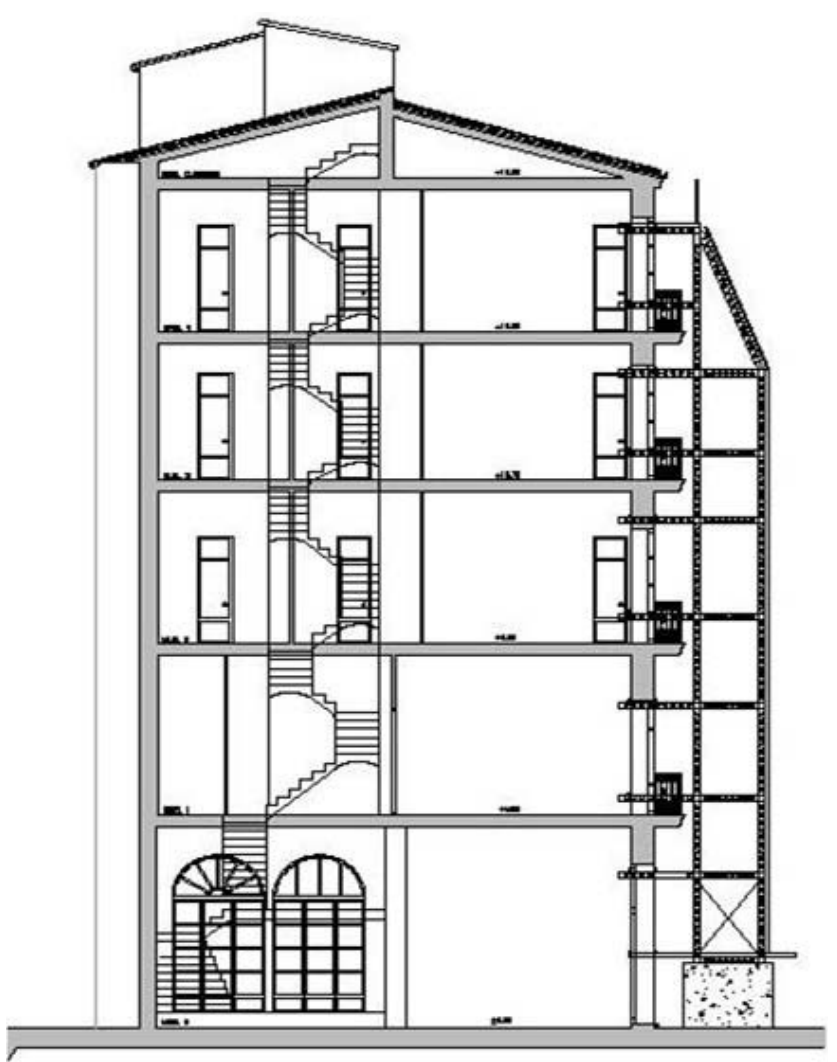

Figura 9. Sección transversal del edificio c/ El Salvador. (Aportado por la propiedad).
Los pares de la $1^{\mathrm{a}}$ crujía apoyan los 7 primeros en el muro de fachada, sin elementos de transición, y en la VIGA 1 (la más próxima a fachada) (Detalle croquis 1, Figura 10), mientras que los 7 restantes apoyan en el muro de fachada y en la VIGA 2 (la que está en un plano inferior). Los pares de la $2^{\mathrm{a}}$ crujía apoyan en la VIGA 2 (Detalle croquis 2, Figura 10) y en el muro que delimita el patio interior. Los pares de la $3^{\text {a }}$ crujía apoyan en la viga 2 (la que está en un plano superior) (Detalle croquis 2, Figura 10) y en otra viga embebida en el muro posterior. Este último apoyo se realiza por medio de unos tacos de madera (Detalle croquis 3, Figura 10).

La madera parece ser de igual tipo y calidad en las crujías 1 y 3, mientras que en la crujía 2 evidencian ser reutilizadas. No se ha realizado una identificación anatómica de la madera empleada en el edificio pero por las características organolépticas de la misma, se estima que se trata de madera de Pinus sylvestris, teniendo en cuenta que la madera empleada en Valencia era traída por el río Turia desde los bosques del Marqués de Moya en la provincia de Cuenca, de pino silvestre y laricio. Es la madera que se conoce como madera de río o pi roig.

\subsection{Estudios realizados}

El estudio realizado partía de dos premisas fundamentales: presentar los resultados en un corto período de tiempo por necesidades de realizar la intervención de manera inmediata y obtener datos de forma rápida y eficaz. Ello restringía el empleo de varias técnicas no destructivas, planteándose una toma de datos como resultado de una inspección visual a fin de tener una clasificación visual y establecer su clase resistente y posterior examen mediante Pilodyn para corroborar los datos anteriormente obtenidos, determinando su contenido de humedad mediante xilohigrómetro. En este caso, el contenido de humedad estaba en el orden del $11 \%$ siendo prácticamente uniforme en los elementos lígneos del edificio, por lo que esta variable no tenía influencia en las distintas mediciones realizadas en los ensayos de penetración.

Las mediciones con Pilodyn (Pd) se realizaron en viguetas desde la cara superior dado que se habían retirado pavimentos y rellenos que completaban los forjados, obteniéndose datos correspondientes a una dirección tangencial, según el siguiente procedimiento: $1^{\mathrm{a}}$ medida, a $25 \mathrm{~cm}$ del paramento interior de los muros, $2^{\mathrm{a}}$ medida y la $3^{\mathrm{a}}$ medida, a $1 \mathrm{~m}$ de las anteriores con respecto al paramento interior o bien al apoyo en las vigas. Si era necesario se realizaban más mediciones intermedias aumentando en razón de $15 \mathrm{~cm}$, hasta definir la extensión del daño; por el contrario, si no se habían detectado alteraciones y las mediciones de penetración estaban dentro de los valores «normales» del conjunto de viguetas, no se realizaba más que uno o dos puntos de lectura. También se tenía la precaución de no incidir en zonas de fendas o de nudos, buscando madera sana y limpia para tener una referencia de «calidad» de la madera empleada. Se concentraron las mediciones en los apoyos por ser las zonas más afectadas por pudrición, aunque en la segunda crujía, donde se localizaban zonas de cocinas, había zonas con pudrición en la longitud de las viguetas.

\subsection{Análisis de los datos obtenidos}

Mediante la inspección visual se definió la clase resistente según la norma UNE 56544:2011. Clasificación visual de la 


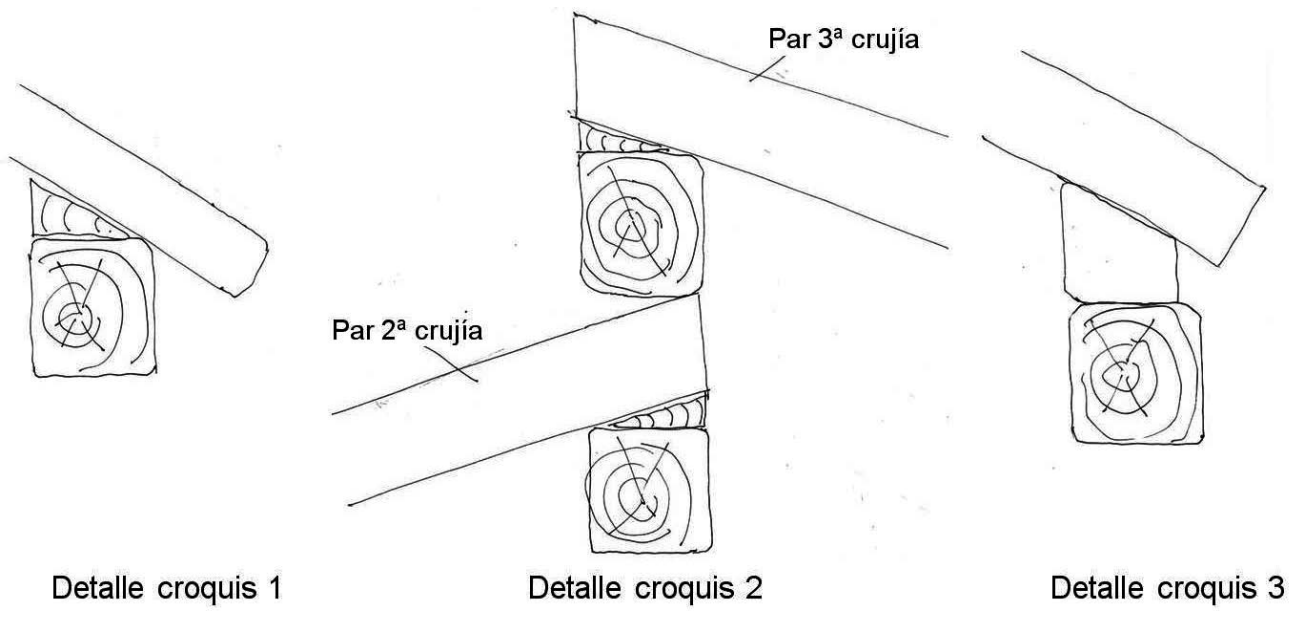

Figura 10. Croquis indicando la forma en la que apoyan los pares en las vigas de cubierta

madera aserrada para uso estructural. Madera de coníferas, siendo ésta C18 (ME-2) (33) y grado único MEG para madera estructural de gruesa escuadría, de grueso superior a $15 \mathrm{~cm}$, con las principales características siguientes: resistencia a flexión, $\mathrm{f}_{\mathrm{m}, \mathrm{k}}, 14 \mathrm{~N} / \mathrm{mm}^{2}$, valor medio de módulo de elasticidad $9 \mathrm{kN} / \mathrm{mm}^{2}$, y densidad media para esta especie de 380 $\mathrm{kg} / \mathrm{m}^{3}$ (32). Las alteraciones del material detectadas, tanto en vigas y viguetas como en pares han sido ataques generalizados de carcoma común y de carcoma grande, y puntualmente pudrición. Es mayor el daño apreciado por pudrición que el producido por otros agentes, siendo menor en este caso, al causado por la carcoma común (Anobium punctatum). Los ataques de carcoma grande (Hylotrupes Bajulus) están generalizados, llegando a tener algunas aristas tanto de viguetas como de pares, profundidades de ataques del orden de 3 a $4 \mathrm{~cm}$, y en los laterales de 1,5 a $3 \mathrm{~cm}$. En algunos elementos, indicados en la toma de datos, se han localizado ataques de termitas, aunque no se han encontrado señales de que éstos estuvieran activos en ese momento.

Los valores de resistencia a la penetración dieron resultados entre 9 y $12 \mathrm{~mm}$ en la madera sana y limpia, mientras que estos valores eran de 14 a $25 \mathrm{~mm}$, e incluso más, si se trataba de definir la extensión de la pudrición en zonas afectadas. Analizando los valores de penetración de $13 \mathrm{~mm}$, que se producen en 28 viguetas de 192 (268 puntos de lectura) con una incidencia de un 10\% de los valores de penetración, se pudo comprobar que, según la inspección visual, correspondían tanto a viguetas de madera sana como a madera con una alteración muy puntual. Éstos no se tuvieron en cuenta para

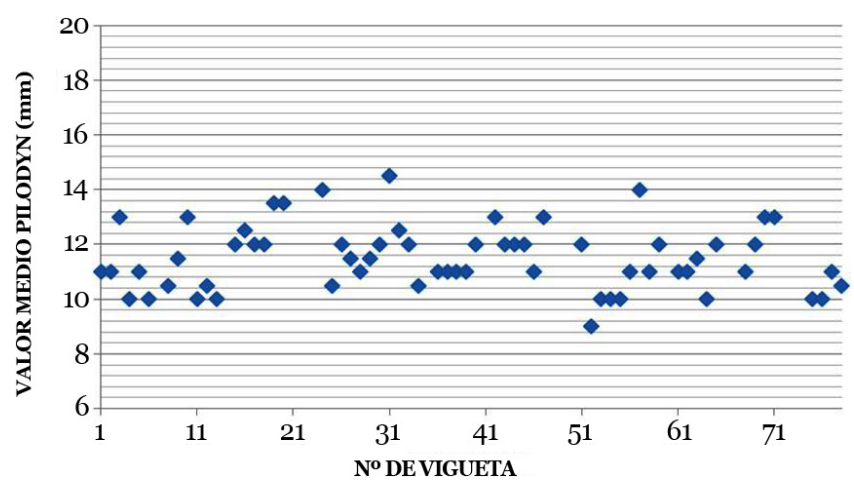

Figura 11. Valores medios de Pd en mm de cada una de las viguetas la consideración de valores medios para madera sana, y por el mismo motivo no podían considerarse para los valores medios de madera alterada.

Los datos obtenidos se volcaron en una hoja de cálculo y se trasladaron a los planos de planta de cada nivel. Un código de colores permitió detectar de manera rápida tanto los valores mayores de penetración, así como las observaciones realizadas durante la inspección visual.

El resumen de los datos de penetración mediante Pilodyn (Pd), considerando valores medios en madera sana por vigueta, de un total de 76, es el que se presenta en la Figura 11.

Considerando los valores de penetración de la varilla indicados, entre 9 y $12 \mathrm{~mm}$ en madera sana, el valor medio de penetración es de 10,96 mm, para 61 viguetas, que se aprecia en la Figura 12. Teniendo en cuenta los resultados de los ensayos de caracterización realizados por Bobadilla (6), en el pino silvestre los datos de Pilodyn eran de $10,78 \mathrm{~mm}$, correspondiendo a valores medios de densidad de $500 \mathrm{~kg} / \mathrm{m}^{3}$ estando, por lo tanto, dentro de los parámetros esperados.

Con respecto al tratamiento de resultados de la madera alterada, y a fin de acotar su extensión se realizaron ensayos de penetración según el procedimiento indicado, que se trasladaron tanto a una hoja de cálculo como a los planos de cada planta, indicando la posición y extensión de zonas afectadas. Sería muy extenso presentar aquí los datos de todas las plantas, incluyéndose solamente aquellos obtenidos en la planta $4^{\mathrm{a}}$, que se refle- 
jan en la Tabla $2^{*}$. Esta tabla reúne tanto los datos observados en la inspección visual, así como los resultados de Pd y H\%.

Según el análisis del conjunto de datos se pudo indicar el estado de la estructura, recomendar las medidas a tomar para su reparación y verificar analíticamente la estructura reduciendo la sección resistente de los elementos afectados por carcoma grande y considerando que tanto cabezas como otras zonas afectadas por pudrición serían reparadas mediante sistema Beta.

El tiempo consumido en el trabajo de campo se redujo considerablemente con respecto a otras inspecciones en las que se emplearon NDT y otros SDT, y los datos obtenidos se consideraron suficientes con respecto al propósito del diagnóstico, cumpliendo con la premisa para su realización en un breve período de tiempo.

\section{CONCLUSIONES}

Los datos de penetración del Pilodyn permiten diferenciar distintas clases resistentes de madera de una misma especie e incluso proponer que algunos sistemas estructurales estén construidos con maderas de especies diferentes. Para ello se han de realizar varias lecturas a lo largo de la pieza, y en zonas en las que no existan defectos o anomalías. Una vez determinadas las cualidades de resistencia a la penetración de una o de varias especies, es posible identificar partes dañadas de los elementos e incluso determinar su extensión, siempre que se trate de alteraciones superficiales.

En el caso de estudio, tras la realización de las operaciones necesarias para el conocimiento de la estructura y del edificio, se ha procedido a la medición de la resistencia a la penetración de la madera mediante Pilodyn una vez se determinado el contenido de humedad de la madera mediante xilohigrómetro. Las dos variables a determinar eran las siguientes:

- Identificar distintas especies o clases de madera empleadas en una estructura

Se ha de tener en cuenta que el tipo de madera que compone los elementos estructurales podrá ser de albura o de duramen, según los despieces que se hayan realizado de la sección escuadrada (apartado 3.1). Por ese motivo, el resultado obtenido será de mayor precisión si se realizan mediciones en todas las caras que queden accesibles, debiéndose señalar la dirección en la que se realiza en ensayo de penetración en cada punto. Las lecturas a realizar son las siguientes:

- En todas las caras accesibles, verificando si la penetración e la aguja será radial o transversal (ver Figura 3)

- En zona de madera sana y limpia

- Identificada la zona afectada, corroborar la alteración que afecta a la pieza y precisar su extensión.

Las zonas que suelen estar más afectadas son los extremos de los elementos lígneos que asientan en las obras de fábrica. En el caso de localización de locales húmedos o en cubiertas, las alteraciones pueden no coincidir con esta patología, pudiéndose manifestar en cualquier punto de los elementos.

Dada la aplicabilidad de este instrumento para el diagnóstico de estructuras de madera en edificios antiguos, parece oportuno proponer una normalización para la realización de este tipo de ensayos.

\section{REFERENCIAS}

(1) Palaia, L., Monfort J., Tormo, S. (2008). Assessment of timber structures in service, by using combined methods of nondestructive testing together with traditional ones. ART2008, The e-Journal \& Exhibition of Nondestructive Testing.

(2) Palaia, L. (2008). Ancient timber structure analysis applying NDT and traditional methods of assessment. First International RILEM Symposium On Site Assessment of Concrete, Masonry and Timber Structures (SACoMaTis 20o8), International RILEM Symposium, Varenna (Italia), RILEM Publications S.A.R.L.

(3) Palaia, L., Tormo, S., López, V., Monfort, J. (2010). NDT assessment of timber structures; Case study: Villa Ivonne, Meliana. Structural Studies, Repairs and Maintenance of Heritage Architecture XII, WIT PRESS, pp. 529-540, doi: http:// dx.doi.org/10.2495/STR110441.

(4) Bobadilla-Maldonado, I., Esteban-Herrero, M., Íñiguez-González, G., Arriaga-Martitegui, F., Ballarín-Montesinos, D., Palacios-Monteagudo, J. (2007). Estimación de la densidad de la madera aserrada de conífera mediante técnicas no destructivas de arranque de tornillos y penetrómetro y su aplicación en la estimación del módulo de elasticidad. Informes de la Construcción, 59(506): 107-116, doi: http://dx.doi.org/10.3989/ic.2007.v59.i506.513.

(5) Arriaga, F., Esteban, M., Relea, E. (2005). Evaluation of the load carrying capacity of large cross section coniferous timber in standing structures. Materiales de Construcción, 55(280): 43-52, doi: http://dx.doi.org/10.3989/mc.2005.v55. i280.205.

(6) Acuña, L., Basterra, L.A., Casado, M., López, G., Ramon-Cueto, G., Relea, E., Martínez, C., González, A. (2011). Aplicación del resistógrafo a la obtención de la densidad y la diferenciación de especies de madera. Materiales de Construcción, 61(303): 451-464, doi: http://dx.doi.org/10.3989/mc.2010.57610.

(7) Daga, S.L. Comercial de ingeniería. Penetrómetro Pilodyn. http://www.dagasl.es/PILODYN.pdf

(8) Hansen C.P. (2002). Application of the Pilodyn in forest tree improvement. Danida Forest Seed Centre, Technical Note $\mathrm{n}^{\mathrm{o}}$ 55, Humlebaek, Denmark.

(9) Taylor, F.W. (1981). Rapid Determination of Southern Pine Specific Gravity with a Pilodyn Tester. Forest Science, 27(1): 59-61.

(10) Hoffmeyer, P. (1978). The Pilodyn instrument as a non-destructive tester of the shock resistance of wood. 4th Nondestructive Testing of Wood. Symposium, pp. 47-66. Vancouver, WA, USA.

\footnotetext{
* Consultar tabla al final del artículo.
} 
(11) Crown, D.J. (1978). Comparison of the Pilodyn and Torsiometer methods for the rapid assessment of wood density in living trees. New Zealand Journal of Forestry Science, 8(3): 384-391.

(12) Wang, T., Aitken, S.N., Rozenberg, P., Carlson, M.R. (1999). Selection for height growth and Pilodyn pin penetration in lodgepole pine: effects on growth traits, wood properties, and their relationships. Canadian Journal of Forest Research, 29(4): 434-445.

(13) Koizumi, A., Takuro, H. (2006). Evaluation of the section modulus for tree-stem cross sections of irregular shape. Journal of Wood Science, 52(3): 213-219, doi: http://dx.doi.org/10.1007/s10086-005-0747-2.

(14) Ishiguri, F., Matsui, R., Iizuka, K., Yokota S., Yoshizawa N. (2008). Prediction of the mechanical properties of lumber by stress-wave velocity and Pilodyn penetration of 36-year-old Japanese larch trees. Holz als Roh- und Werkstoff, 66(4): 275-280, doi: http://dx.doi.org/10.1007/s00107-008-0251-7.

(15) Korzeniowski, A., Dzbeński, W. (1994). Predicting mechanical properties of coniferous structural timber from impact hardness. Proceedings of the First European Symposium on Non Destructive Evaluation of Wood, pp.62-76. University of Sopron, Hungary.

(16) Giuriani, E., Gubana, A. (1993). A penetration test to evaluate wood decay and its application to the Loggia monument. RILEM Materials and Structures, 26(1): 8-14, doi: http://dx.doi.org/10.1007/BFo2472232.

(17) Giuriani, E., Gubana, A. (1994). A penetration test to evaluate wood decay. Proceedings of the First European Symposium on Non Destructive Evaluation of Wood, pp. 21-23. University of Sopron, Hungary.

(18) Ronca, P., Gubana, A. (1998). Mechanical characterization of wooden structures by means of an in situ penetration test. Construction and Building Materials, 12(4): 233-243, doi: http://dx.doi.org/10.1016/So950-0618(97)00080-9.

(19) ISO 3133 Wood- Determination of ultimate strength in static bending

(20) UNE-EN 408:2004 Estructuras de madera. Madera aserrada y madera laminada encolada para uso estructural. Determinación de algunas propiedades físicas y mecánicas.

(21) UNE-EN 13183:2002; err 2003; Ac 2004. I-II Contenido de humedad de una pieza de madera aserrada.

(22) Piazza, M., Riggio, M. (2008). Visual Strength-grading and NDT of timber in traditional structures. Journal of Building Appraisal, 3: 267-296, doi: http://dx.doi.org/10.1057/jba.2008.4.

(23) Piazza, M., Riggio M., Tomasi, R., Giongo, I. (2010). Comparison of In Situ and Laboratory Testing for the characterization of Old Timber Beams before and after Intervention. Advanced Materials Research, 133-134: 1101-1106, doi: http:// dx.doi.org/10.4028/www.scientific.net/AMR.133-134.1101.

(24) Riggio, M., Piazza, M. (2011). Hardness Test. In Situ Assessment of Structural Timber, 7: 87-97. RILEM State of the Art Reports, doi: http://dx.doi.org/10.1007/978-94-007-0560-9_10.

(25) Görlacher, R. (1987). Non destructive testing of Wood: an in-situ method for determination of density. Holz als Roh-und Werkstoff, 45(7): 273-278, doi: http://dx.doi.org/10.1007/BF02608673.

(26) Branco, J.M., Piazza, M., Cruz, P.J.S. (2010). Structural analysis of two King-post timber trusses: Non-destructive evaluation and load-carrying tests. Construction and Building Materials, 24(3): 371-383, doi: http://dx.doi.org/10.1016/j. conbuildmat.2009.08.025.

(27) Schueremans, L. (2009). Assessing historic timber roof structures. Methodology and case studies from a Belgian viewpoint. Conference Series on Theoretical and Practical Issues on Built Heritage Conservation, Tusnad.

(28) UNE 56544:2011. Clasificación visual de la madera aserrada para uso estructural. Madera de coníferas.

(29) Pla y Rave, E. (2003). Manual del maderero (ed. Facsímil de la ed. de 1897, Madrid), pp. 191 y s.s. Editorial Maxtor.

(30) Piqueras-Haba, J., Sanchis-Deusa, C. (2001). El transporte fluvial de madera en España. Cuadernos de Geografía, (6970): 127-162.

(31) Palaia-Pérez, L. (2011). Los sistemas constructivos empleados por los maestros carpinteros de los siglos XV al XVII en Valencia. CIMAD 11- $1^{\circ}$ Congresso Ibero-LatinoAmericano da Madeira na Construção, Coimbra, Portugal.

(32) Llopis, A., Perdigón, L., Taberner, F. (2004). Cartografía histórica de la ciudad de Valencia. Volumen 1 (1608-1929). Universitat Politècnica de València, Valencia.

(33) Arriaga-Martitegui, F., Íñiguez-González, G., Esteban-Herrero, M., Bobadilla-Maldonado I. (2007). Madera aserrada de gran escuadría para uso estructural y sus particularidades. Informes de la Construcción, 59(506): 29-41, doi: http:// dx.doi.org/10.3989/ic.2007.v59.i506.505. 


\begin{tabular}{|c|c|c|c|c|c|c|c|c|c|c|c|c|c|c|c|c|c|c|c|c|c|c|c|c|c|c|c|c|c|c|c|}
\hline & 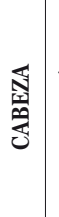 & 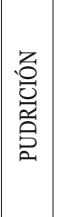 & 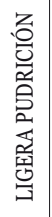 & 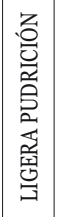 & 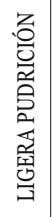 & 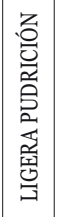 & 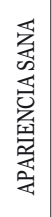 & 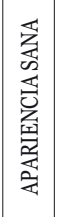 & 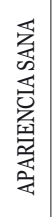 & 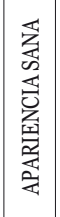 & 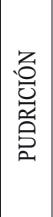 & 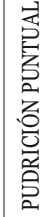 & 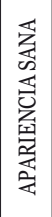 & & & 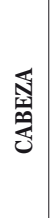 & 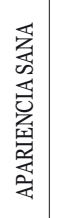 & 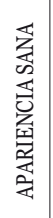 & $\begin{array}{l}\text { 总 } \\
\text { 营 } \\
\text { 总 }\end{array}$ & 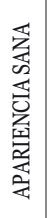 & 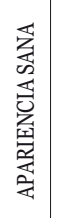 & 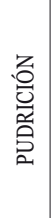 & & & 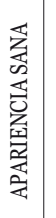 & 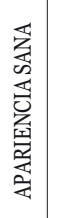 & 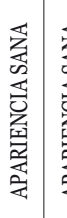 & & 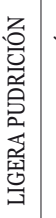 & 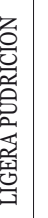 & \\
\hline 童 & छ్ & & & & & & & & & & & & & & 音 & 吾 & & & & & & & 音 & छ్ & & & & & & & \\
\hline 产 & 馬 & $\cong$ & $\Phi$ & 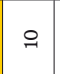 & $\approx$ & $\cong$ & 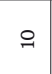 & & & & & & & & $\stackrel{\ddot{m}}{\vec{\theta}}$ & 馬 & $\nexists$ & & & & & & 㟥 & ఏ & & & & & 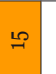 & & \\
\hline 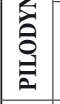 & 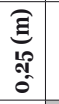 & $\cong$ & 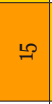 & $\Re$ & $\cong$ & $\sigma$ & $\Rightarrow$ & $\Rightarrow$ & $\therefore$ & $\Rightarrow$ & $a$ & $\Re$ & $\approx$ & & 言 & 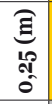 & $\Xi$ & $\exists$ & $\Rightarrow$ & $\cong$ & $\cong$ & 움 & 言 & $\begin{array}{l}\overline{\mathrm{E}} \\
\mathrm{g} \\
\mathrm{h} \\
\mathrm{d}\end{array}$ & $\because$ & 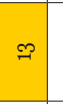 & 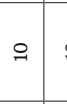 & $\approx$ & 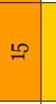 & 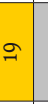 & $\bowtie$ \\
\hline 필 & 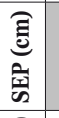 & ळ & $R$ & $\mathfrak{2}$ & $\delta$ & 주 & $R$ & if & 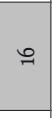 & $\Re$ & $\stackrel{\infty}{\wedge}$ & $i$ & สิ & & 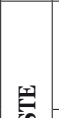 & 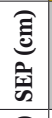 & $F$ & $\stackrel{\infty}{0}$ & $E$ & 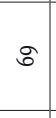 & 8 & $R$ & 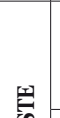 & 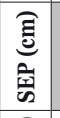 & ○ & 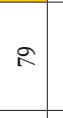 & $\infty$ & ผู่ & $\infty$ & $i$ & \\
\hline 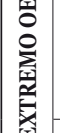 & 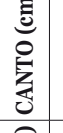 & a & d & A & A & A & A & t & ส & t & d & A & d & & 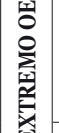 & 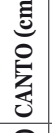 & ส & A & A & a & a & a & 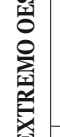 & 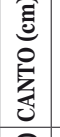 & ส & t & a & I & T & ส & \\
\hline 焉 & 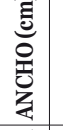 & 战 & & & & & & 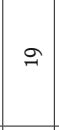 & ล & $\cong$ & 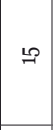 & $\exists$ & $\Re$ & & 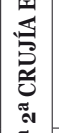 & 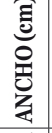 & $\nsubseteq$ & $\approx$ & $\cong$ & $\approx$ & $a$ & $\Rightarrow$ & 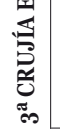 & 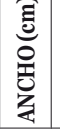 & \pm & $\Rightarrow$ & 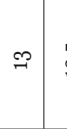 & 幽 & 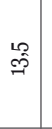 & \pm & \\
\hline & 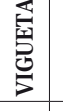 & - & 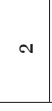 & $\infty$ & $\sigma$ & 10 & 0 & 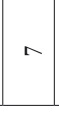 & $\infty$ & $a$ & 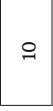 & $\Rightarrow$ & $\approx$ & & o & 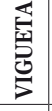 & $r$ & 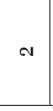 & $\infty$ & $\sigma$ & 10 & 0 & $\stackrel{\sigma}{+}$ & 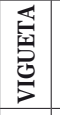 & $\rightarrow$ & N & $\infty$ & $\sigma$ & 10 & 6 & \\
\hline & 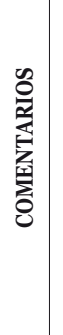 & & & & & 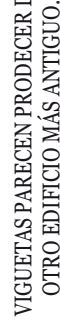 & & 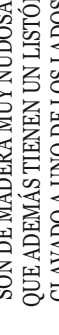 & & & & & & & & $\begin{array}{l}\text { 是 } \\
\text { 产 } \\
\text { 产 }\end{array}$ & & & 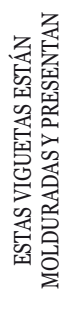 & & & & & 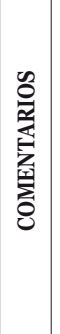 & & & & & & & \\
\hline & 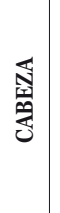 & 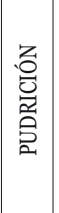 & 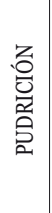 & 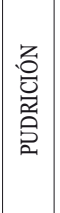 & 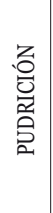 & 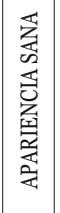 & 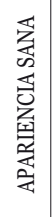 & 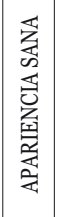 & 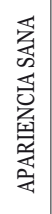 & $\begin{array}{l}\text { 总 } \\
\text { 息 } \\
\text { 宫 }\end{array}$ & 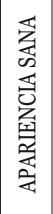 & 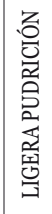 & 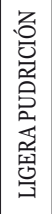 & & & 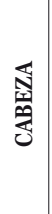 & 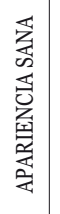 & 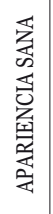 & 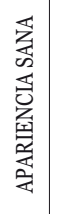 & 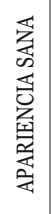 & 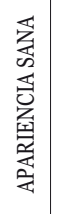 & 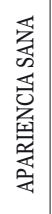 & & 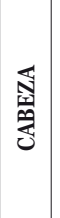 & 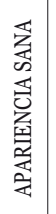 & 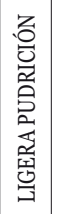 & 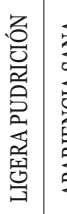 & 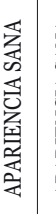 & 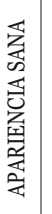 & 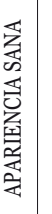 & \\
\hline 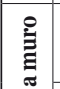 & 吾 & & i & & $\Rightarrow$ & & & & $\Rightarrow$ & & & & & & 蒿 & 吾 & & & & & & & 音 & 囦 & & & & & & & \\
\hline 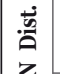 & $\widehat{\mathrm{E}}$ & & & & & & & & & & & & & & 㝘 & & & & & & & & $\begin{array}{l}\dot{\vec{D}} \\
\overrightarrow{0}\end{array}$ & 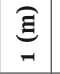 & & $\stackrel{p}{2}$ & & & & & \\
\hline 商 & 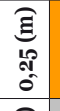 & $\hat{a}$ & $\Rightarrow$ & $\vec{\pi}$ & $g$ & $\Rightarrow$ & $\sigma$ & $\Rightarrow$ & $\vec{\Xi}$ & i & $\Rightarrow$ & $\exists$ & 㐭 & & 惫 & 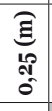 & $\Rightarrow$ & 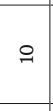 & 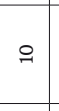 & $a$ & $\cong$ & $\Rightarrow$ & 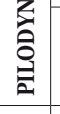 & $\begin{array}{c}\mathrm{g} \\
\mathrm{g} \\
\mathrm{L} \\
\mathrm{\delta} \\
\end{array}$ & $\cong$ & 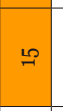 & $\Rightarrow$ & $\approx$ & $\exists$ & $\Phi$ & \\
\hline & $\begin{array}{l}\text { 产 } \\
\text { 畺 } \\
\end{array}$ & गे & $\mathfrak{2}$ & 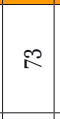 & 6 & $R$ & $\infty$ & $\infty$ & 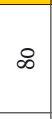 & I & $\stackrel{\infty}{\wedge}$ & $\stackrel{\mathfrak{R}}{\mathfrak{R}}$ & N & A & & 产 & 7 & $\infty$ & $E$ & 8 & $\stackrel{8}{0}$ & 2 & & 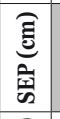 & $\circ$ & $\infty$ & 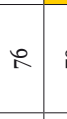 & $\curvearrowright$ & 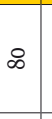 & $\curvearrowright$ & $q$ \\
\hline 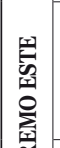 & 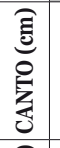 & A & ส & A & ส & A & A & A & A & A & A & A & A & & 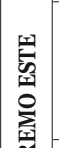 & 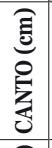 & A & a & ส & A & ส & A & 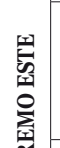 & 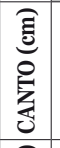 & ส & A & A & d & A & d & \\
\hline 穿 & 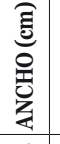 & $\cong$ & $\mathscr{D}$ & $\stackrel{\infty}{\sim}$ & & & & $\Rightarrow$ & & & & & & & 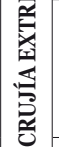 & 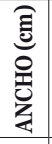 & $\mathscr{\sim}$ & $\cong$ & $\approx$ & $\approx$ & $\sigma$ & $\Rightarrow$ & 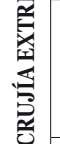 & 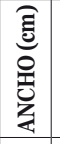 & 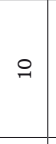 & $\stackrel{2}{2}$ & $\mathscr{D}$ & $\exists$ & $\stackrel{20}{=}$ & $\cong$ & \\
\hline 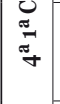 & $\begin{array}{l}\text { do } \\
\overrightarrow{\vec{t}} \\
\end{array}$ & $\Rightarrow$ & $\Rightarrow$ & $\Rightarrow$ & $\Rightarrow$ & $\Rightarrow$ & $\Rightarrow$ & $\Rightarrow$ & $\Rightarrow$ & $\Rightarrow$ & $\Rightarrow$ & $\Rightarrow$ & $\Rightarrow$ & & $\begin{array}{l}0 \\
\text { of } \\
\text { of } \\
\text { of }\end{array}$ & 蓠 & $\Rightarrow$ & $\Rightarrow$ & $\Rightarrow$ & $\Rightarrow$ & $\Rightarrow$ & $\Rightarrow$ & 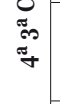 & 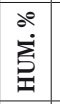 & $\Rightarrow$ & $\Rightarrow$ & $\Rightarrow$ & $\Rightarrow$ & $\Rightarrow$ & $\Rightarrow$ & \\
\hline & 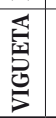 & - & a & $\infty$ & $\sigma$ & 10 & 0 & $n$ & $\infty$ & $a$ & 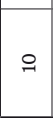 & $\Rightarrow$ & $\nsubseteq$ & & & $\begin{array}{l}\text { 预 } \\
\text { 包 } \\
\end{array}$ & $\rightarrow$ & N & $\infty$ & $\sigma$ & L & 0 & & 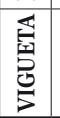 & -1 & a & $\infty$ & $\nabla$ & 10 & 6 & \\
\hline
\end{tabular}

